TRANSACTIONS OF THE

AMERICAN MATHEMATICAL SOCIETY

Volume 354, Number 11, Pages 4303-4312

S 0002-9947(02)03081-7

Article electronically published on July 2, 2002

\title{
KÄHLER-EINSTEIN METRICS FOR SOME QUASI-SMOOTH LOG DEL PEZZO SURFACES
}

\author{
CAROLINA ARAUJO
}

\begin{abstract}
Recently Johnson and Kollár determined the complete list of anticanonically embedded quasi-smooth log del Pezzo surfaces in weighted projective 3-spaces. They also proved that many of those surfaces admit a KählerEinstein metric, and that some of them do not have tigers.

The aim of this paper is to settle the question of the existence of KählerEinstein metrics and tigers for those surfaces for which the question was left open. In order to do so, we will use techniques developed earlier by Nadel, Demailly and Kollár.
\end{abstract}

\section{INTRODUCTION}

Recently Johnson and Kollár determined the complete list of anticanonically embedded quasi-smooth log del Pezzo surfaces in weighted projective 3-spaces [JK]. Then, using techniques developed earlier by Demailly and Kollár [DK], they proved that many of those surfaces admit a Kähler-Einstein metric, and that some of them do not have tigers. Later Boyer, Galicki and Nakamaye showed the existence of Kähler-Einstein metrics for a general surface of degree 16 in weighted projective space with weights 1, 3,5, 8 [BGN1]. Moreover, it follows from their (BGN2], Corollary 5.3) that a general surface of degree 18 in weighted projective space with weights 2,3,5,9 also admits a Kähler-Einstein metric. These were two of the log del Pezzo surfaces for which the question was left open by Johnson and Kollár.

The aim of this paper is to settle the question of the existence of Kähler-Einstein metrics and tigers for those surfaces in [JK] for which the question was still open. In order to show the existence of Kähler-Einstein metrics for these surfaces, we will use a criterion described in the work of Nadel [Na] and Demailly and Kollár [DK].

\section{Anticanonically embedded quasi-Smooth log del Pezzo surfaces IN WEIGHTED PROJECTIVE 3 -SPACES}

For positive integers $q_{i}$ let $\mathbb{P}\left(q_{0}, q_{1}, q_{2}, q_{3}\right)$ denote the weighted projective 3-space with weights $q_{0}, q_{1}, q_{2}, q_{3}$. (See [Dol] or [Fle for basic definitions and results.) When there is no ambiguity, we abbreviate $\mathbb{P}=\mathbb{P}\left(q_{0}, q_{1}, q_{2}, q_{3}\right)$. We always assume that any 3 of the $q_{i}$ are relatively prime. Let $x_{0}, x_{1}, x_{2}, x_{3}$ denote the corresponding weighted projective coordinates. $\mathbb{P}\left(q_{0}, q_{1}, q_{2}, q_{3}\right)$ can be viewed as the quotient

$$
\mathbb{C}^{4} \backslash\{0\} / \mathbb{C}^{*}\left(q_{0}, q_{1}, q_{2}, q_{3}\right),
$$

Received by the editors December 12, 2001.

2000 Mathematics Subject Classification. Primary 14Q10, 32Q20.

Partial financial support was provided by CNPq (Conselho Nacional de Desenvolvimento Científico e Tecnológico - Brazil). 
where $\mathbb{C}^{*}\left(q_{0}, q_{1}, q_{2}, q_{3}\right)$ denotes the $\mathbb{C}^{*}$-action on $\mathbb{C}^{4} \backslash\{0\}$ given by

$$
\left(z_{0}, z_{1}, z_{2}, z_{3}\right) \mapsto\left(t^{q_{0}} z_{0}, t^{q_{1}} z_{1}, t^{q_{2}} z_{2}, t^{q_{3}} z_{3}\right) .
$$

The action of $\mathbb{C}^{*}$ on $\mathbb{C}^{4} \backslash\{0\}$ induces the action $\mathbb{Z}_{q_{i}}\left(q_{j}, q_{k}, q_{l}\right)$ on $V_{i}=\left\{z_{i}=1\right\} \subset \mathbb{C}^{4}$ which, after identifying $V_{i}$ with $\mathbb{C}^{3}$, can be written as

$$
\left(z_{j}, z_{k}, z_{l}\right) \mapsto\left(\xi^{q_{j}} z_{j}, \xi^{q_{k}} z_{k}, \xi^{q_{l}} z_{l}\right),
$$

where $\xi$ is a primitive $q_{i}$ th root of unity. Hence the affine chart $U_{i} \subset \mathbb{P}$ where $x_{i} \neq 0$ can be identified with

$$
\mathbb{C}^{3} / \mathbb{Z}_{q_{i}}\left(q_{j}, q_{k}, q_{l}\right)
$$

These are called the orbifold charts. Write $p_{i}: \mathbb{C}^{3} \rightarrow U_{i}$ for the natural projection.

$\mathbb{P}\left(q_{0}, q_{1}, q_{2}, q_{3}\right)$ has an index $q_{i}$ singularity at $P_{i}=\left(x_{j}=0\right)_{j \neq i}$, and an index $\left(q_{i}, q_{j}\right)$ singularity along the line $\left(x_{k}=0\right)_{k \neq i, j}$.

For every $d \in \mathbb{Z}$ there is a rank 1 sheaf $\mathcal{O}_{\mathbb{P}}(d)$, and the sections of $\mathcal{O}_{\mathbb{P}}(d)$ consist of the homogeneous polynomials $f\left(x_{0}, x_{1}, x_{2}, x_{3}\right)$ of weighted degree $d$.

Let $X=X_{d}$ be a member of $\left|\mathcal{O}_{\mathbb{P}}(d)\right|$. If $X$ does not contain any of the singular lines of $\mathbb{P}$, then the adjunction formula

$$
\left.\left.K_{X} \cong \mathcal{O}_{\mathbb{P}}\left(K_{\mathbb{P}}+X\right)\right|_{X} \cong \mathcal{O}_{\mathbb{P}}\left(d-\left(q_{0}+q_{1}+q_{2}+q_{3}\right)\right)\right|_{X}
$$

holds.

A log del Pezzo surface is a projective surface with quotient singularities such that its anticanonical class is ample.

If $X$ does not contain any of the singular lines, then, by the adjunction formula, its anticanonical class is ample if and only if $d<q_{0}+q_{1}+q_{2}+q_{3}$. Here we are interested in the case when $d$ is as large as possible, i.e., $d=q_{0}+q_{1}+q_{2}+q_{3}-1$. In this case we say that $X$ is anticanonically embedded in $\mathbb{P}\left(q_{0}, q_{1}, q_{2}, q_{3}\right)$.

We say that $X$ is quasi-smooth if the pre-image of $X$ under the quotient map

$$
\mathbb{C}^{4} \backslash\{0\} \rightarrow \mathbb{C}^{4} \backslash\{0\} / \mathbb{C}^{*}\left(q_{0}, q_{1}, q_{2}, q_{3}\right)=\mathbb{P}\left(q_{0}, q_{1}, q_{2}, q_{3}\right)
$$

is smooth.

We write conditions on the weights $q_{0}, q_{1}, q_{2}, q_{3}$ for the general surface $X_{d} \subset$ $\mathbb{P}\left(q_{0}, q_{1}, q_{2}, q_{3}\right)$ of degree $d=q_{0}+q_{1}+q_{2}+q_{3}-1$ to be quasi-smooth, and for the adjunction formula to hold. These conditions then imply that the general $X_{d}$ is an anticanonically embedded quasi-smooth log del Pezzo surface:

Conditions 2.1. For $X_{d}$ to be quasi-smooth (see [Fle], 8.5):

1. for every $i$ there is $a j$ and a monomial $x_{i}^{m_{i}} x_{j}$ of degree $d$;

2. for all distinct $i$ and $j$, either there is a monomial $x_{i}^{b_{i}} x_{j}^{b_{j}}$ of degree $d$, or there are monomials $x_{i}^{c_{i}} x_{j}^{c_{j}} x_{k}$ and $x_{i}^{d_{i}} x_{j}^{d_{j}} x_{l}$ of degree $d$, with $k$ and $l$ distinct.

For $X_{d}$ not to contain any of the singular lines of $\mathbb{P}$ (which implies that the adjunction formula holds):

3. for all distinct $i$ and $j$ such that $\left(q_{i}, q_{j}\right)>1$, there is a monomial $x_{i}^{b_{i}} x_{j}^{b_{j}}$ of degree $d$.

Notation 2.2. Given a monomial $M$, and a polynomial $f$, we write $M \in f$ if the coefficient of $M$ in $f$ is nonzero. 


\section{Tigers And the EXISTEnCE OF KäHLER-Einstein METRICS}

We start this section by giving some definitions from the log category. We refer to $[\mathrm{KM}]$ for a detailed introduction.

Definition 3.1. Let $X$ be a normal surface, and $D$ a $\mathbb{Q}$-divisor on $X$ such that $m\left(K_{X}+D\right)$ is Cartier for some $m>0$. Let $f: Y \rightarrow X$ be a proper birational morphism from a smooth surface $Y$. Then there is a unique $\mathbb{Q}$-divisor $\sum e_{i} E_{i}$ on $Y$ such that

$$
K_{Y} \equiv f^{*}\left(K_{X}+D\right)+\sum e_{i} E_{i} \text { and } f_{*} \sum e_{i} E_{i}=-D .
$$

We say that the pair $(X, D)$ is canonical (resp. klt, resp. log canonical) if $e_{i} \geq 0$ (resp. $e_{i}>-1$, resp. $e_{i} \geq-1$ ) for every $f$ and every $i$.

Remark 3.2. If $(X, D)$ and $\left(X, D^{\prime}\right)$ are both canonical (resp. klt, resp. log canonical), then so is $\left(X, \alpha D+(1-\alpha) D^{\prime}\right)$ for any $0 \leq \alpha \leq 1$. This is a very easy result, but it will be very useful in our applications.

Definition 3.3 ([KMcK $]$ ). Let $X$ be a normal surface. A tiger on $X$ is an effective $\mathbb{Q}$-divisor $D \equiv-K_{X}$ such that $(X, D)$ is not klt.

We use the following sufficient condition for the existence of a Kähler-Einstein metric on log del Pezzo surfaces:

Theorem 3.4 ([Na], $[\mathrm{DK}])$. Let $X$ be a log del Pezzo surface. If there is an $\epsilon>0$ such that $\left(X, \frac{2+\epsilon}{3} D\right)$ is klt for every effective $\mathbb{Q}$-divisor $D \equiv-K_{X}$, then $X$ admits a Kähler-Einstein metric.

In order to prove the existence of a Kähler-Einstein metric (resp. the nonexistence of tigers) for a surface $X_{d} \subset \mathbb{P}\left(q_{0}, q_{1}, q_{2}, q_{3}\right)$, we will show that, for all effective $\mathbb{Q}$-divisors $D \equiv-\frac{2+\epsilon}{3} K_{X}\left(\operatorname{resp} D \equiv K_{X}\right),(X, D)$ is klt. The definition of a klt pair $(X, D)$, however, involves understanding all resolutions of singularities of $(X, D)$. Instead, we will use the following multiplicity conditions:

Conditions $3.5([\mathrm{JK}])$. Let $X=X_{d}$ be a hypersurface of weighted degree $d$ in $\mathbb{P}\left(q_{0}, q_{1}, q_{2}, q_{3}\right)$, and assume that $X$ is quasi-smooth and has only isolated (quotient) singularities. Write

$$
p_{i}: \mathbb{C}^{3} \rightarrow U_{i}=\left(x_{i} \neq 0\right) \cong \mathbb{C}^{3} / \mathbb{Z}_{q_{i}}\left(q_{j}, q_{k}, q_{l}\right)
$$

for the natural projections to the orbifold charts. For an effective $\mathbb{Q}$-divisor $D$ on $X,(X, D)$ is klt provided that the following 3 conditions hold:

0 . $D$ does not contain any irreducible component with coefficient $\geq 1$;

1. for every smooth point $P \in X$, mult $_{P} D \leq 1$;

2. for every singular point $P \in X \cap U_{i}$, mult $_{Q} p_{i}^{*} D \leq 1$, where $p_{i}(Q)=P$.

In our applications, condition (3.5.0) can be verified right away. To check the other 2 conditions, we will bound the multiplicities above by suitable intersection numbers.

3.1. The Smooth Points. Let $P \in X^{0}$, the nonsingular locus of $X$. Suppose we can find a positive integer $l$ such that, for any effective $\mathbb{Q}$-divisor $D \equiv-K_{X}$, there is a divisor $F \in\left|\mathcal{O}_{X}(l)\right|$ such that:

1. $F=F^{\prime}+B$, where $F^{\prime}$ is an effective Weil divisor, and $B$ is nef;

2. $F^{\prime}$ passes through $P$; 
3. $F^{\prime}$ does not contain any irreducible component of $D$.

Then we can intersect $D$ with $F^{\prime}$, and obtain:

$$
\text { mult }_{P} D \leq\left(D \cdot F^{\prime}\right) \leq(D \cdot F)=\frac{l d}{q_{0} q_{1} q_{2} q_{3}} .
$$

We would like this bound to be $\leq 1$ in order to verify Condition 3.51. Unfortunately in most of the cases we cannot choose an $l$ that works for all $P \in X^{0}$. Instead, we will find an $l$ that works for a dense open set of $X^{0}$, say $X^{0} \backslash C$, and then show by other methods that mult $_{P} D \leq 1$ for $P \in C \cap X^{0}$.

Lemma 3.6. Let $\mathbb{P}=\mathbb{P}\left(q_{0}, \ldots, q_{n}\right)$ denote the weighted projectice $n$-space with weights $q_{0}, \ldots, q_{n}$. Let $l$ be a positive integer such that for every $i \neq 0$ there are at least 2 distinct monomials in $H^{0}\left(\mathbb{P}, \mathcal{O}_{\mathbb{P}}(l)\right)$ of the form $x_{0}^{\alpha} x_{i}^{\beta}$. Then, for every $P \in \mathbb{P} \backslash\left(x_{0}=0\right)$, and every divisor $D$ on $\mathbb{P}$, there is a divisor $F \in\left|\mathcal{O}_{\mathbb{P}}(l)\right|$ that can be written as $F=F^{\prime}+a\left(x_{0}=0\right)$, where $a$ is a nonnegative integer, and $F^{\prime}$ is an effective Weil divisor passing through $P$ and not containing any irreducible component of $D$.

Proof. We choose from each irreducible component $D_{j}$ of $D, D_{j} \not \subset\left(x_{0}=0\right)$, a point $Q_{j} \in D_{j} \backslash\left(x_{0}=0\right)$ (later we will impose a further condition on the $Q_{j}$ ). We shall prove that there is a polynomial $F_{j} \in H^{0}\left(\mathbb{P}, \mathcal{O}_{\mathbb{P}}(l)\right)$ that vanishes at $P$ but not at $Q_{j}$. We then choose $F$ to be a suitable linear combination of the $F_{j}$.

Let $Q=Q_{j}$. Fix representations $P=\left(1: a_{1}: \ldots: a_{n}\right)$ and $Q=\left(1: b_{1}\right.$ : $\left.\cdots: b_{n}\right)$ in weighted projective coordinates (notice that these representations are not unique). If $a_{i}=0 \neq b_{i}$ or $a_{i} \neq 0=b_{i}$ for some $i$, then it is easy to find a polynomial in $H^{0}\left(\mathbb{P}, \mathcal{O}_{\mathbb{P}}(l)\right)$ that vanishes at $P$ but not at $Q$. So we assume that for every $i$, either $a_{i}=0=b_{i}$ or $a_{i} \neq 0 \neq b_{i}$.

For each $i \neq 0$ fix $x_{0}^{\alpha_{i}} x_{i}^{\beta_{i}}$ and $x_{0}^{\gamma_{i}} x_{i}^{\delta_{i}}$ distinct monomials in $H^{0}\left(\mathbb{P}, \mathcal{O}_{\mathbb{P}}(l)\right)$, and assume $\delta_{i}>\beta_{i}$. Define:

$$
H_{i}=a_{i}^{\delta_{i}} x_{0}^{\alpha_{i}} x_{i}^{\beta_{i}}-a_{i}^{\beta_{i}} x_{0}^{\gamma_{i}} x_{i}^{\delta_{i}} \in H^{0}\left(\mathbb{P}, \mathcal{O}_{\mathbb{P}}(l)\right) .
$$

Then $H_{i}(P)=0$, and $H_{i}(Q)=0$ if and only if $b_{i}=\zeta_{i} a_{i}$, where $\zeta_{i}^{\delta_{i}-\beta_{i}}=1$.

Therefore, if $Q$ is outside the finite set $\left\{\left(1: \zeta_{1} a_{1}: \ldots: \zeta_{n} a_{n}\right) \mid \zeta_{i}^{\delta_{i}-\beta_{i}}=1\right\}$ (and we can certainly impose that condition when we choose the $Q_{j}$ above), we get a polynomial in $H^{0}\left(\mathbb{P}, \mathcal{O}_{\mathbb{P}}(l)\right)$ that vanishes at $P$ but not at $Q$.

Now we will use Lemma 3.6] to find a linear system $\left|\mathcal{O}_{X_{d}}(l)\right|$ on $X_{d}$ that will give us a bound for mult $_{P} D$, at least for $P \in X_{d} \cap\left(x_{0} \neq 0\right)$.

Corollary 3.7. Let $X=X_{d} \subset \mathbb{P}\left(q_{0}, q_{1}, q_{2}, q_{3}\right)$ be an anticanonically embedded quasi-smooth log del Pezzo surface. Let $\pi_{3}: X \rightarrow \mathbb{P}=\mathbb{P}\left(q_{0}, q_{1}, q_{2}\right)$ denote the projection from $P_{3}=(0,0,0,1)$. Assume $\pi_{3}$ has only finite fibers. Let l be a positive integer such that, for $i=1,2$, there are at least 2 monomials in $H^{0}\left(\mathbb{P}, \mathcal{O}_{\mathbb{P}}(l)\right)$ of the form $x_{0}^{\alpha} x_{i}^{\beta}$.

Then, for every $P \in X^{0} \backslash\left(x_{0}=0\right)$ and every effective $\mathbb{Q}$-divisor $D \equiv-K_{X}$, there is a divisor $F \in\left|\mathcal{O}_{X}(l)\right|$ that can be written as $F=F^{\prime}+a\left(x_{0}=0\right)$, where a is a nonnegative integer, and $F^{\prime}$ is an effective Weil divisor passing through $P$ and not containing any irreducible component of $D$. Hence mult $_{P} D \leq \frac{l d}{q_{0} q_{1} q_{2} q_{3}}$.

Proof. Set $P^{\prime}=\pi_{3}(P) \in \mathbb{P}$, and $D^{\prime}=\pi_{3 *} D$. By the lemma, there is a divisor $E \in\left|\mathcal{O}_{\mathbb{P}}(l)\right|$ that can be written as $E=E^{\prime}+a\left(x_{0}=0\right)$, where $a$ is a nonnegative 
integer, and $E^{\prime}$ is an effective Weil divisor passing through $P^{\prime}$ and not containing any irreducible component of $D^{\prime}$. Since $\pi_{3}$ has only finite fibers, we can take $F$ to be $\pi_{3}^{*} E \in \pi_{3}^{*}\left|\mathcal{O}_{\mathbb{P}}(l)\right| \subset\left|\mathcal{O}_{X}(l)\right|$.

Now we have to deal with the smooth points of $X$ in $\left(x_{0}=0\right)$.

Lemma 3.8. Let $X=X_{d} \subset \mathbb{P}\left(q_{0}, q_{1}, q_{2}, q_{3}\right)$ be an anticanonically embedded quasismooth log del Pezzo surface. Assume that $C=X \cap\left(x_{0}=0\right)$ is irreducible and smooth outside the singular locus of $X$. If $d \leq q_{1} q_{2} q_{3}$, then for every effective $\mathbb{Q}$ divisor $D \equiv-K_{X}$ and every $P \in X^{0} \cap C$ we have mult $_{P} D \leq 1$.

Proof. Fix $P \in X^{0} \cap C$. Since $C$ is smooth outside the singular locus of $X$, mult $_{P} C=1$. Given $D \equiv-K_{X}$, write $D=\alpha C+\left(1-\alpha q_{0}\right) D^{\prime}$, where $0 \leq \alpha \leq 1 / q_{0}$, $D^{\prime} \equiv-K_{X}$, and $C$ is not contained in the support of $D^{\prime}$. Then

$$
\text { mult }_{P} D^{\prime} \leq\left(C \cdot D^{\prime}\right) \leq \frac{d}{q_{1} q_{2} q_{3}} \leq 1
$$

and hence mult $_{P} D \leq \alpha+\left(1-q_{0} \alpha\right) \leq 1$.

Now we put these results together to obtain conditions on the weights $q_{i}$ for every effective $\mathbb{Q}$-divisor $D \equiv-K_{X_{d}}$ to have multiplicity $\leq 1$ at the smooth points of $X_{d}$.

Lemma 3.9. Let $X=X_{d} \subset \mathbb{P}\left(q_{0}, q_{1}, q_{2}, q_{3}\right)$ be an anticanonically embedded quasismooth log del Pezzo surface. Let $f$ be the defining polynomial of $X_{d}$ in $\mathbb{P}\left(q_{0}, q_{1}\right.$, $\left.q_{2}, q_{3}\right)$. Assume that:

1. the curve $C=X \cap\left(x_{0}=0\right)$ is irreducible and smooth outside the singular locus of $X$;

2. $x_{3}^{a} \in f$ for some $a \in \mathbb{Z}, a \geq 1$;

3. $d \leq \min \left\{q_{1}, q_{2}\right\} \cdot q_{3}$.

Then mult $_{P} D \leq 1$ for every smooth point $P \in X$ and every $\mathbb{Q}$-divisor $D \equiv-K_{X}$.

Proof. Let $D$ be a $\mathbb{Q}$-divisor such that $D \equiv-K_{X}$.

Conditions 3.9.1 and 3.9. 3 together with Lemma 3.8 imply that mult $_{P} D \leq 1$ for every smooth point $P \in C$.

Condition 3.9.2 implies that the projection $\pi_{3}$ has only finite fibers. Thus the hypotheses of Corollary 3.7 are fulfilled for $l=q_{0} \cdot \max \left\{q_{1}, q_{2}\right\}$. (Indeed, assuming $q_{1} \leq q_{2}$, we have that $\left.x_{0}^{q_{2}}, x_{0}^{q_{2}-q_{1}} x_{1}^{q_{0}}, x_{2}^{q_{0}} \in\left|\mathcal{O}_{X}\left(q_{0} q_{2}\right)\right|.\right)$ Thus we get that mult $_{P} D \leq \frac{l d}{q_{0} q_{1} q_{2} q_{3}} \leq 1$ for every $P \in X^{0} \backslash C$.

\subsection{The Singular Points.}

Estimate 3.10. Let $X=X_{d} \subset \mathbb{P}\left(q_{0}, q_{1}, q_{2}, q_{3}\right)$ be a surface of degree $d$ defined by the polynomial $f$. Let $P \in X \cap U_{i}$ be a singular point, and let $p_{i}:(S, Q) \rightarrow(X, P)$ be a local orbifold chart. Assume that some monomial of the form $x_{i}^{a} x_{l}^{b} \in f$, where $\{i, j, k, l\}=\{0,1,2,3\}$. Then, for every effective $\mathbb{Q}$-divisor $D \equiv-K_{X}$,

$$
\operatorname{mult}_{Q} p_{i}^{*} D \leq \frac{d}{\min \left(q_{j}, q_{k}\right) \cdot q_{l}} .
$$

Proof. Consider the linear system $\left|p_{i}^{*}\left(x_{j}^{q_{k}}\right), p_{i}^{*}\left(x_{k}^{q_{j}}\right)\right| \subset p_{i}^{*}\left|\mathcal{O}_{X}\left(q_{j} q_{k}\right)\right|$. 
The assumption that $x_{i}^{a} x_{l}^{b} \in f$ implies that $\left(x_{j}=x_{k}=0\right) \not \subset X$. Thus the above linear system does not have any fixed components. So we can intersect a general member of it with $p_{i}^{*} D$, obtaining:

$$
\operatorname{mult}_{Q} p_{i}^{*} D \leq \frac{q_{i}}{\min \left(q_{j}, q_{k}\right)}\left(D \cdot \mathcal{O}_{X}\left(q_{j} q_{k}\right)\right)=\frac{d}{\min \left(q_{j}, q_{k}\right) \cdot q_{l}} .
$$

When this bound is $>1$ but $\leq 2$, we can look closer at what happens near $Q$, that is, we can blow-up this point and then use Shokurov's Inversion of Adjunction:

Theorem 3.11 ( $\underline{\mathrm{KM}}, 5.50)$. Let $S$ be a smooth surface, $Q \in S$, and $B$ be an effectice $\mathbb{Q}$-divisor such that mult ${ }_{Q} B \leq 2$. Let $\pi: S^{\prime} \rightarrow S$ be the blow-up of $Q$ with exceptional divisor $E$. If $\left.\left(\pi_{*}^{-1}(B)\right)\right|_{E}$ is a sum of points, all with coefficients $\leq 1$, then $(S, B)$ is $\log$ canonical at $Q$.

\section{The Main Result}

In this section we answer the question of the existence of Kähler-Einstein metrics and tigers for the six surfaces in [JK] for which the question was still unsolved. In Theorem 4.1 below, the existence of a Kähler-Einstein metric was not known for the first two families of surfaces, $X_{10} \subset \mathbb{P}(1,2,3,5)$ and $X_{15} \subset \mathbb{P}(1,3,5,7)$. As for the remaining ones, it was not known if they could have tigers.

Theorem 4.1. Let $X_{d} \subset \mathbb{P}\left(q_{0}, q_{1}, q_{2}, q_{3}\right)$ denote any anticanonically embedded quasi-smooth log del Pezzo surface in the weighted projective 3-space with weights $q_{0}, q_{1}, q_{2}, q_{3}$. Then:

1. $X_{10} \subset \mathbb{P}(1,2,3,5)$ admits a Kähler-Einstein metric.

2. $X_{15} \subset \mathbb{P}(1,3,5,7)$ admits a Kähler-Einstein metric provided that the coeffcient of the monomial $x_{1} x_{2} x_{3}$ in the equation of $X_{15}$ is nonzero.

3. $X_{18} \subset \mathbb{P}(2,3,5,9)$ does not have a tiger.

4. $X_{25} \subset \mathbb{P}(3,5,7,11)$ does not have a tiger.

5. $X_{28} \subset \mathbb{P}(3,5,7,14)$ does not have a tiger.

6. $X_{36} \subset \mathbb{P}(3,5,11,18)$ does not have a tiger.

Remarks 4.2. 1. The nonexistence of tigers on the last four families of surfaces implies that these surfaces admit a Kähler-Einstein metric.

2. $\left(x_{0}=0\right)$ is a tiger on the surfaces in (4.11) and (4.1.2).

3. In (4.1.2), if the coefficient of $x_{1} x_{2} x_{3}$ in the equation of $X_{15}$ is zero, then the criterion of Theorem 3.4 does not apply. Indeed, $c \cdot\left(x_{0}=0\right)$ is not klt for any $c>8 / 15$. However, it is possible that even in this case, $X_{15}$ admits a KählerEinstein metric.

Proof. Let $f$ denote the defining polynomial of $X=X_{d}$ in $\mathbb{P}\left(q_{0}, q_{1}, q_{2}, q_{3}\right)$.

1. $X_{10} \subset \mathbb{P}(1,2,3,5)$.

The polynomial $f$ is a linear combination of the monomials in $\left|\mathcal{O}_{\mathbb{P}(1,2,3,5)}(10)\right|$. Since $X$ is quasi-smooth, we can apply Condition 2.1.1 with $i=1,2,3$ and conclude that $x_{1}^{5}, x_{0} x_{2}^{3}, x_{3}^{2} \in f$.

Let $C=X \cap\left(x_{0}=0\right)$. It is easy to see that the only monomials of the form $x_{1}^{a_{1}} x_{2}^{a_{2}} x_{3}^{a_{3}}$ in $\left|\mathcal{O}_{\mathbb{P}(1,2,3,5)}(10)\right|$ are $x_{1}^{5}, x_{1}^{2} x_{2}^{2}, x_{1} x_{2} x_{3}$, and $x_{3}^{2}$. Hence, after rescaling, 
the equations for $C$ in $\mathbb{P}(1,2,3,5)$ become:

$$
\begin{aligned}
& x_{0}=0, \\
& x_{1}^{5}+a x_{1}^{2} x_{2}^{2}+b x_{1} x_{2} x_{3}+x_{3}^{2}=x_{1}^{5}+\left(\alpha x_{1} x_{2}+x_{3}\right)\left(\beta x_{1} x_{2}+x_{3}\right)=0 .
\end{aligned}
$$

We see that $C$ is irreducible and smooth outside the singular locus of $X$.

Let $D \equiv-K_{X}$ be an effective $\mathbb{Q}$-divisor. We shall show that $\left(X, \frac{7}{10} D\right)$ is $\log$ canonical. This is more than enough to apply Theorem 3.4 and then conclude that $X$ admits a Kähler-Einstein metric.

First let us consider the smooth points of $X$. As we have seen above, $C$ is irreducible and smooth on $X^{0}$, and $x_{3}^{2} \in f$. So we can apply Lemma 3.9 to conclude that $D$ has multiplicity $\leq 1$ at the smooth points of $X$. Therefore, by Condition $3.51,\left(X, \frac{7}{10} D\right)$ is $\log$ canonical (in fact klt) at the smooth points of $X$.

Now let us consider the singular points of $X$. These are the singular points of $\mathbb{P}(1,2,3,5)$ that lie on $X$ (since $X$ is quasi-smooth). The singular points of $\mathbb{P}(1,2,3,5)$ are $P_{1}=(0: 1: 0: 0), P_{2}=(0: 0: 1: 0)$, and $P_{3}=(0: 0: 0: 1)$. Since $x_{1}^{5}, x_{3}^{2} \in f$, the points $P_{1}$ and $P_{3}$ do not lie on $X$. Since there is no pure power of $x_{2}$ in $\left|\mathcal{O}_{\mathbb{P}(1,2,3,5)}(10)\right|, P_{2} \in X$, and it is the only singular point of $X$. Let

$$
p_{2}:\left(\mathbb{C}^{3}, Q_{2}=0\right) \rightarrow\left(U_{2}=\left(x_{2} \neq 0\right), P_{2}\right)
$$

denote the natural projection to the orbifold chart.

Write $D=\lambda C+(1-\lambda) D^{\prime}$, with $0 \leq \lambda \leq 1, D^{\prime} \equiv-K_{X}$, and $C$ not contained in the support of $D^{\prime}$. By Remark 3.2 it is enough to show that both $\left(X, \frac{7}{10} C\right)$ and $\left(X, \frac{7}{10} D^{\prime}\right)$ are $\log$ canonical.

From the equations (11) above, we see that mult $_{Q_{2}} p_{2}^{*} C=2$, and thus we can use Shokurov's inversion of adjunction. Let the notation be as in Theorem 3.11 We have 2 cases: If $\alpha \neq \beta$, then $p_{2}^{*} C$ has a simple node at $Q_{2}$, and $\left(\pi_{*}^{-1}\left(p_{2}^{*} C\right)\right) \mid E$ is a sum of 2 points, each with coefficient 1 . In this case, $(X, C)$ is log canonical at $P_{2}$. If $\alpha=\beta$, then $p_{2}^{*} C$ has only one tangent direction at $Q_{2}$, and $\left(\pi_{*}^{-1}\left(p_{2}^{*} C\right)\right) \mid E$ is a single point, with coefficient 2 . In this case, $(X, C)$ is not log canonical at $P_{2}$. However, using $([\overline{\mathrm{Kol}}], 8.15)$ and $([\overline{\mathrm{Kol}}], 5.20)$, we see that $\left(X, \frac{7}{10} C\right)$ is $\log$ canonical at $P_{2}$.

Now for $D^{\prime}$ we have:

$$
\text { mult }_{Q_{2}} p_{2}^{*} D^{\prime} \leq \frac{1}{2}\left(p_{2}^{*} D^{\prime} \cdot p_{2}^{*} C\right) \leq \frac{3}{2}\left(D^{\prime} \cdot C\right)=\frac{1}{2},
$$

and hence $\left(X, \frac{7}{10} D^{\prime}\right)$ is $\log$ canonical at $P_{2}$.

2. $X_{15} \subset \mathbb{P}(1,3,5,7)$.

Let $C=X \cap\left(x_{0}=0\right) \equiv-K_{X}$. The only monomials of the form $x_{1}^{a_{1}} x_{2}^{a_{2}} x_{3}^{a_{3}}$ in $\left|\mathcal{O}_{\mathbb{P}(1,3,5,7)}(15)\right|$ are $x_{1}^{5}, x_{1} x_{2} x_{3}$, and $x_{2}^{3}$. Condition 2.1.1 for $i=1,2$ implies that $x_{1}^{5}, x_{2}^{3} \in f$. If $x_{1} x_{2} x_{3} \notin f$, then, after rescaling, the equations of $C$ in $\mathbb{P}(1,3,5,7)$ become:

$$
x_{0}=0, \quad x_{1}^{5}+x_{2}^{3}=0,
$$

and $\left(X, \frac{2}{3} C\right)$ is not klt. (To see this, notice that $\left(S=p_{3}^{-1}(X), p_{3}^{*}\left(\frac{2}{3} C\right)\right.$ ) is not klt by $([\mathrm{Kol}], 8.15)$, and then apply $([\mathrm{Kol}], 5.20)$.) In this case, the criterion of Theorem 3.4 cannot be applied. From now on we assume that $x_{1} x_{2} x_{3} \in f$. Then, after rescaling, the equations of $C$ in $\mathbb{P}(1,3,5,7)$ become:

$$
x_{0}=0, \quad x_{1}^{5}+x_{1} x_{2} x_{3}+x_{2}^{3}=0 .
$$

One sees easily that $C$ is irreducible and smooth on $X^{0}$. 
Let $D \equiv-K_{X}$ be an effective $\mathbb{Q}$-divisor. We shall show that $(X, D)$ is $\log$ canonical. This is more than enough to apply Theorem 3.4 .

As we have seen above, $x_{2}^{3} \in f$, and $C$ is irreducible and smooth outside the singular locus of $X$. Thus we can apply Lemma 3.9 for $X_{15} \subset \mathbb{P}(1,3,7,5)$, and conclude that $(X, D)$ is log canonical at the smooth points of $X$.

$P_{3}=(0: 0: 0: 1)$ is the only singular point of $X$. Write $D=\alpha C+(1-\alpha) D^{\prime}$, with $0 \leq \alpha \leq 1, D \equiv-K_{X}$, and $C$ not contained in the support of $D^{\prime}$. We just need to show that both $(X, C)$ and $\left(X, D^{\prime}\right)$ are $\log$ canonical at $P_{3}$.

From the equations (2) above, we see that $p_{3}^{*} C$ has a simple node at $Q_{3}$. So mult $_{Q_{3}} p_{3}^{*} C=2$, and we can use Shokurov's inversion of adjunction. Let the notation be as in Theorem 3.11. Then $\left.\left(\pi_{*}^{-1}\left(p_{3}^{*} C\right)\right)\right|_{E}$ is a sum of 2 points, each with coefficient 1 . We conclude that $(X, C)$ is $\log$ canonical at $P_{3}$.

Now for $D^{\prime}$ we have:

$$
\text { mult }_{Q_{3}} p_{3}^{*} D^{\prime} \leq \frac{1}{2}\left(p_{3}^{*} D^{\prime} \cdot p_{3}^{*} C\right) \leq \frac{7}{2}\left(D^{\prime} \cdot C\right)=\frac{1}{2},
$$

and hence $\left(X, D^{\prime}\right)$ is log canonical at $P_{3}$.

3. $X_{18} \subset \mathbb{P}(2,3,5,9)$.

Condition [2.1.1 for $i=2,3$ implies that $x_{1} x_{2}^{3}, x_{3}^{2} \in f$. After rescaling, the equations for $C=X \cap\left(x_{0}=0\right)$ can be written as:

$$
x_{0}=0, \quad x_{3}^{2}+x_{1} x_{2}^{3}+a x_{1}^{6}+b x_{3}=0 .
$$

$C$ is irreducible and smooth outside the singular locus of $X$.

Let $D \equiv-K_{X}$ be an effective $\mathbb{Q}$-divisor. We will show that $(X, D)$ is klt.

We apply Lemma 3.9 to conclude that $D$ is klt at the smooth points of $X$.

The singular points of $X$ are $P_{2}=(0: 0: 1: 0)$ and $P_{\lambda}=(0: 1: 0: \lambda)$, where $\lambda$ satisfies $\lambda^{2}+b \lambda+a=0$. Estimate 3.10 shows that mult $Q_{Q_{2}} p_{2}^{*} D \leq 1$ (here take $(i, j, k, l)=(2,0,1,3))$, and mult $_{Q_{\lambda}} p_{1}^{*} D \leq 1$ (here take $\left.(i, j, k, l)=(1,0,2,3)\right)$. Thus $D$ is klt at the singular points of $X$.

4. $X_{25} \subset \mathbb{P}(3,5,7,11)$.

Condition 2.11 for $i=1,2,3$ implies that $x_{1}^{5}, x_{2}^{2} x_{3}, x_{0} x_{3}^{2} \in f$. After rescaling we can write the equations for $C=X \cap\left(x_{0}=0\right) \in\left|\mathcal{O}_{X}(3)\right|$ as:

$$
x_{0}=0, \quad x_{1}^{5}+x_{2}^{2} x_{3}=0 .
$$

We see that $C$ is irreducible and smooth outside the singular locus of $X$.

Let $D \equiv-K_{X}$ be an effective $\mathbb{Q}$-divisor.

We apply Lemma 3.9 to $X_{25} \subset \mathbb{P}(3,7,11,5)$, and conclude that $D$ is klt at the smooth points of $X$.

The singular points of $X$ are $P_{0}=(1: 0: 0: 0), P_{2}=(0: 0: 1: 0)$ and $P_{3}=$ $(0: 0: 0: 1)$. Estimate 3.10 takes care of $P_{0}$ and $P_{2}($ take $(i, j, k, l)=(0,1,2,3)$ and $(2,0,1,3)$ respectively), but it only gives that mult $_{Q_{3}} p_{3}^{*} D \leq 25 / 21$ (here take $(i, j, k, l)=(3,0,1,2))$. We can improve this bound:

From the equations (3) above, we see that $p_{3}^{*} C \cong\left(y_{1}^{5}+y_{2}^{2}=0\right) \subset \mathbb{C}_{\left(y_{1}, y_{2}\right)}^{2}$, and so mult $_{Q_{3}} p_{3}^{*} C=2$. Write $D=\alpha C+(1-3 \alpha) D^{\prime}$, where $0 \leq \alpha \leq 1 / 3, D^{\prime} \equiv-K_{X}$, and $C$ is not contained in the support of $D^{\prime}$. Then:

$$
\text { mult }_{Q_{3}} p_{3}^{*} D^{\prime} \leq \frac{1}{2}\left(p_{3}^{*} C \cdot p_{3}^{*} D^{\prime}\right) \leq \frac{11}{2}\left(\mathcal{O}_{X}(3) \cdot \mathcal{O}_{X}(1)\right)=\frac{5}{14} .
$$

So mult $_{Q_{3}} p_{3}^{*} D \leq 2 / 3$, and we have taken care of all singular points.

5. $X_{28} \subset \mathbb{P}(3,5,7,14)$. 
Condition 2.11 for $i=1,3$ implies that $x_{0} x_{1}^{5}, x_{3}^{2} \in f$. For $i=2$, it implies that either $x_{2}^{4} \in f$ or $x_{2}^{2} x_{3} \in f$. After rescaling, the equations for $C=X \cap\left(x_{0}=0\right)$ become:

$$
x_{0}=0, \quad x_{3}^{2}+a x_{2}^{2} x_{3}+b x_{2}^{4}=\left(x_{3}+\alpha x_{2}^{2}\right)\left(x_{3}+\beta x_{2}^{2}\right)=0 .
$$

We see that $C$ is the union of 2 irreducible components.

Let $D \equiv-K_{X}$ be an effective $\mathbb{Q}$-divisor.

We can apply Corollary 3.7 with $l=21$ (indeed, $x_{3}^{2} \in f$ implies that $\pi_{3}$ has finite fibers, and $\left.x_{0}^{7}, x_{0}^{2} x_{1}^{3}, x_{2}^{3} \in\left|\mathcal{O}_{\mathbb{P}(3,5,7,14)}(21)\right|\right)$, and conclude that mult $_{P} D \leq 2 / 5$ for every smooth point $P \in X \backslash\left(x_{0}=0\right)$. Since $C$ is reducible, we cannot apply Lemma [3.9. Instead we apply Corollary [3.7 again with $x_{0}$ and $x_{2}$ interchanged, and $l=35$ ( $\pi_{3}$ has finite fibers, and $\left.x_{2}^{5}, x_{1}^{7}, x_{2}^{2} x_{0}^{7} \in\left|\mathcal{O}_{\mathbb{P}(3,5,7,14)}(35)\right|\right)$. This shows that for every smooth point $P \in X \backslash\left(x_{2}=0\right)$, mult $_{P} D \leq 2 / 3$. Since $X \cap\left(x_{0}=\right.$ $0) \cap\left(x_{2}=0\right)=\left\{P_{1}\right\}$, this takes care of all smooth points of $X$.

The singular points of $X$ are $P_{0}=(1: 0: 0: 0), P_{1}=(0: 1: 0: 0)$ and $P_{\lambda}=(0: 0: 1: \lambda)$, where $\lambda$ satisfies $\lambda^{2}+a \lambda+b=0$. Estimate 3.10 shows that Condition 3.52 for the singular points is satisfied.

6. $X_{36} \subset \mathbb{P}(3,5,11,18)$.

Condition 2.1.1 for $i=1,3$ implies that $x_{1}^{5} x_{2}, x_{3}^{2} \in f$. After rescaling, we can write the equations for $C$ as:

$$
x_{0}=0, \quad x_{1}^{5} x_{2}+x_{3}^{2}=0 .
$$

We see that $C$ is irreducible and smooth outside the singular locus of $X$.

Let $D \equiv-K_{X}$ be an effective $\mathbb{Q}$-divisor.

Lemma 3.9 shows that $D$ is klt at the smooth points of $X$.

The singular points of $X$ are $P_{1}=(0: 1: 0: 0), P_{2}=(0: 0: 1: 0)$ and $P_{\lambda}=(1: 0: 0: \lambda)$, where $\lambda$ satisfies $\lambda^{2}+a \lambda+b=0$. Here $a$ and $b$ are the coefficients of $x_{0}^{6} x_{3}$ and $x_{0}^{12}$ in $f$ respectively, and we still assume that the coefficient of $x_{3}^{2}$ in $f$ is 1 . Estimate 3.10 shows that Condition 3.5.2 for the singular points is satisfied.

\section{ACKNOWLEDGEMENTS}

I would like to thank J. Kollár for very useful comments and corrections. I would also like to thank the Rényi Institute of Mathematics for the hospitality during the conference on Higher Dimensional Varieties and Rational Points - September 2001, when part of this paper was written.

\section{REFERENCES}

[BGN1] C. P. Boyer, K. Galicki and M. Nakamaye: Sasakian-Einstein Structures on $9 \#\left(S^{2} \times S^{3}\right)$. Preprint DG/0102181 (2001).

[BGN2] C. P. Boyer, K. Galicki and M. Nakamaye: On the Geometry of Sasakian-Einstein 5manifolds. Preprint DG/0012047 (2001).

[DK] J.-P. Demailly and J. Kollár: Semi-continuity of complex singularity exponents and Kähler-Einstein metrics on Fano orbifolds. Preprint AG/9910118 (1999), Ann. Scient. École Norm. Sup. Paris 34 (2001), 525-556. MR 2002e:32032

[Dol] I. Dolgachev: Weighted projective varieties, in Group actions and vector fields, Springer Lecture Notes in Math., vol. 956, (1982) 34-71. MR 85g:14060

[Fle] A. R. Fletcher: Working with weighted complete intersections, in Explicit birational geometry of 3-folds, A. Corti and M. Reid, eds., Cambridge Univ. Press. (2000) 101-173. MR 2001f: 14004 
[JK] J. M. Johnson and J. Kollár: Kähler-Einstein metrics on log del Pezzo surfaces in weighted projective 3-spaces. Preprint AG/0008129 (2000), Ann. Inst. Fourier (Grenoble) 51 (2001), 69-79. MR 2002b:32041

[KMcK] S. Keel and J. McKernan: Rational curves on quasi-projective surfaces. Mem. Amer. Math. Soc. 140 (1999), no. 669. MR 99m:14068

[Kol] J. Kollár: Singularities of pairs, Algebraic Geometry, Santa Cruz, 1995, Proc. Sympos. Pure Math. vol. 62, Amer. Math. Soc., (1997) 221-287. MR 99m:14033

$[\mathrm{KM}] \quad$ J. Kollár and S. Mori: Birational geometry of algebraic varieties, Cambridge Univ. Press (1998). MR 2000b:14018

[Na] A. M. Nadel: Multiplier ideal sheaves and Kähler-Einstein metrics of positive scalar curvature, Annals of Math. 132, (1990) 549-596. MR 92d:32038

Mathematics Department, Princeton University, Princeton, New Jersey 08544

E-mail address: caraujo@math.princeton.edu 